Supplement Issue 1: International Tehran Breast Cancer Congress (TBCC9)

\title{
The Relationship Between Reproductive Risk Factors for Breast Cancer and Tumor Molecular Subtypes
}

DOI: $10.21859 / \mathrm{mci}-\mathrm{supp}-81$

\author{
Nafissi ${ }^{1}$, Akbari $\mathrm{ME}^{2}$, Ziaee $\mathrm{F}^{2}$, Hoseini $\mathbf{M}^{1}$, Negahi A ${ }^{1}$, Faraji $\mathbf{M}^{1}$, \\ Seyed Hamzeh Mosavy ${ }^{2, *}$ \\ ${ }^{1}$ Iran University of Medical Science, Tehran, Iran \\ ${ }^{2}$ Cancer Research Center, Shahid Beheshti University of Medical Sciences, Tehran, \\ Iran \\ * Corresponding author: Seyed Hamzeh Mosavy, Cancer Research Center, Shahid \\ Beheshti University of Medical Sciences, Tehran, Iran.E-mail: s.hamzah.mousavie@ \\ gmail.com
}

\section{Keywords: \\ Breast Cancer \\ Tumor Molecular \\ Reproductive Risk}

\begin{abstract}
Introduction: Due to wide clinical differences in various pathological types of breast cancer and also close association between disease prognosis and molecular subtypes of the tumor, it has been recently suggested a relationship between traditional risk factors and molecular subtypes of breast cancer. Hence, the present study aimed to assess the association of reproductive risk factors for breast cancer with tumor molecular subtypes.

Materials and Methods: This bi-center cross-sectional study was performed on 800 consecutive women with known breast cancer who referred to two great referral hospitals as the Comprehensive Cancer Centers in Tehran between 2006 and 2016. The baseline information related to reproductive risk profiles as well as pathological tumor diagnosis and molecular subtypes determined using immune-histochemical analysis by immune-staining for ER, PR, and HER2 molecules were collected by reviewing the hospital recorded files. Results: Of 800 samples included for immune-histochemical analysis, 314 (39.3\%) were positive for Luminal A subtype, 107 (13.4\%) for Luminal B subtype, 153 (19.1\%) for HER-2 over expression, and 226 (28.3\%) for triple negative subtype. Among all reproductive risk factors initially assessed, young age was associated with HER-2 over expression pattern, more tumor size with luminal B subtype, lower age at pregnancy with luminal A subtype, lower number of gravid with triple negative pattern, the history of abortion with luminal B subtype, and shorter duration of breastfeeding with triple negative subtypes. Conclusions: Each molecular subtype of breast cancer in our population can be specifically associated with specific reproductive risk factors.
\end{abstract}

